\title{
Computers in Clinical Cardiology
}

\section{Imaging of Coronary Artery Fistulas by Multidetector Computed Tomography: Is Multidetector Computed Tomography Sensitive?}

\author{
Fehmi Kacmaz, m.D., ${ }^{*}$ Nilgun Isiksalan Ozbulbul, M.D., ${ }^{\dagger}$ Omer Alyan, M.D., ${ }^{*}$ Orhan Maden, m.D., ${ }^{*}$ Ahmet Duran \\ Demir, ${ }^{*}$ Ramazan Atak, ${ }^{*}$ Kubilay Senen, ${ }^{*}$ Ali Riza Erbay, ${ }^{*}$ Yucel Balbay, ${ }^{*}$ Tulay Olcer, ${ }^{\dagger}$ Erdogan Ilkay ${ }^{\ddagger}$ \\ *Cardiology Clinics, Turkish Society of Cardiology; ${ }^{\dagger}$ Department of Radiology, Turkiye Yuksek Ihtisas Hospital, Turkish \\ Society of Radiology; ${ }^{\ddagger}$ Department of Cardiology, Ozel Mesa Hospital, Ankara, Turkey
}

\section{Summary}

Background: Coronary angiography is the gold standard for diagnosing coronary artery fistulas (CAFs). Multidetector computed tomography (MDCT) is a recently developed imaging technique for detecting coronary artery stenosis, coronary artery anomalies, and coronary artery fistulas and their courses.

Objective: We aimed to determine accuracy or sensitivity of MDCT in patients having CAF.

Method: We evaluated 13 patients with 15 CAFs detected earlier by coronary angiography. MDCT was carried out on all patients and the results were compared with coronary angiography, following which, sensitivity of MDCT was detected.

Results: Eleven of $15 \mathrm{CAFs}$ were shown on MDCT and the overall sensitivity of MDCT was found to be $73 \%$. Seven of 8 CAFs that coursed between two vascular structures were detected and the sensitivity of MDCT

This manuscript has been accepted as a poster presentation at the second Annual Congress on Update in Cardiology and Cardiovascular Surgery, and the abstract was published in the Clinical Cardiology journal.

Address for reprints:

Fehmi Kacmaz, M.D.

Bingol Devlet Hastanesi

Kardiyoloji klinigi

12100 Duzagac-Bingol

Ankara, Turkey

e-mail: kacmazfehmi@superonline.com/

drfehmikacmaz@yahoo.com

Received: April 18, 2007

Accepted with revision: August 21, 2007

Published online in Wiley InterScience

(www.interscience.wiley.com).

DOI: $10.1002 /$ clc. 20286

(C) 2008 Wiley Periodicals, Inc. in this group was found to be $87 \%$. However, the sensitivity of $58 \%$ of MDCT in patients with fistula coursing between coronary arteries and cardiac chambers was found.

Conclusion: Although coronary angiography is the gold standard diagnostic test for detection of CAF, MDCT may be an alternative test, especially, for CAF coursing between vascular structures, to detect origin, course, and the drainage site of fistula through its excellent spatial resolution and ability to show relationship of anatomic structures.

Key words: coronary artery fistula, coronary angiography, multidetector computed tomography

Clin. Cardiol. 2008; 31: 41-47.

(C) 2008 Wiley Periodicals, Inc.

\section{Introduction}

Coronary artery fistula $(\mathrm{CAF})$ is a rare anomaly in which a communication is present between a coronary artery and either a cardiac chamber or another vascular structure. This has been observed in $0.3 \%$ to $0.8 \%$ of patients who underwent coronary angiography. ${ }^{1,2}$ The CAF arises from the right coronary artery (RCA) more often than from the left coronary artery (LCA) and arises from both RCA and LCA in approximately 5\% of patients. ${ }^{3}$ More than $90 \%$ of fistulas drain into the systemic venous side of the circulation. ${ }^{4}$ Multidetector computed tomography (MDCT) is a recently developed imaging technique to detect coronary artery stenosis, an anomalous vessel of the coronary artery, and CAF. There are rare case reports of CAF detected by MDCT. However, the accuracy or sensitivity of MDCT in patients having CAF has not been determined before. We described diagnostic sensitivity of MDCT in a series of 13 patients with 15 CAFs. This is the first study as far as we know in current literature. 


\section{Materials and Methods}

\section{Patients}

Between June 2005 and June 2006, a total of 7,854 consecutive patients underwent coronary angiography and 13 patients were incidentally found to have CAFs. All patients were admitted to hospital with complaints of angina and/or dyspnea. Patients who had atrial fibrillation were excluded. Metoprolol $(50-100 \mathrm{mg}$ ) was given orally $90-120 \mathrm{~min}$ prior to the MDCT scan to obtain adequate heart rate. Multidetector computed tomography was carried out on all patients who had CAF detected earlier by coronary angiography. All patients were orally informed about the study which was started after obtaining approval of patients who gave written informed consent, and the study protocol was approved by the Institutional Review Board. To detect sensitivity of MDCT, its results were evaluated by two experienced radiologist and one cardiologist who were unaware about study protocol. Finally, we determined diagnostic sensitivity of MDCT in patients in whom coronary artery fistula was detected.

\section{Multidetected Computed Tomography Image and Data Analysis}

Patients with angiographically documented coronary artery fistulas underwent 16-slice computed tomography (CT) coronary angiography. Medications were not discontinued throughout the angiographic and MDCT studies. MDCT (Light Speed 16, General Electric, Milwaukee, Wisc., USA) was performed 3 to 5 days after coronary angiography. All image acquisitions were performed in the supine position during inspiratory breathhold preceded by mild hyperventilation and inhalation of oxygen $(2-3 \mathrm{~L} / \mathrm{min})$ for $5 \mathrm{~min}$. First, noncontrast localization scan was performed that yielded an anteroposterior view of the chest; it was used to position the imaging volume for coronary artery imaging, which extended from the carina to $1 \mathrm{~cm}$ below the diaphragmal face of the heart. In a second step, a bolus of $30 \mathrm{~mL}$ of contrast agent (Iohexol, Ultravist 350, Schering AG, Berlin, Germany) was injected intravenously at $4 \mathrm{~mL} / \mathrm{second}$ via an 18-gauge catheter placed in the antecubital vein. After a delay of 10 seconds a sequence of 10 axial images at the level of the carina was acquired with an interval of 2 seconds between subsequent images. From the time interval between contrast agent injection and acquisition of the images with peak attenuation in the aortic root, the contrast agent transit time was determined. In a third step, the contrast agent $(130 \mathrm{~mL})$ was injected into the antecubital vein at $4 \mathrm{~mL} / \mathrm{second}$, and the CT scan was initiated with a delay according to the previously determined contrast agent transit time. The dataset for coronary artery visualization was acquired $(16 \times 0.625 \mathrm{~mm}$ detector configuration, table feed $3 \mathrm{~mm} /$ rotation, pitch 0.3 , gantry rotation time $500 \mathrm{~ms}$, tube voltage of $120 \mathrm{kV}$ ).

After acquisition of the raw spiral data, retrospective ECG-synchronized slice were reconstructed from five diastolic data-sets centered at 30, 40, 50, 60 and $70 \%$ of the R-R interval. All image data-sets concerning the presence of motion artifacts and each coronary artery were inspected. The dataset that contained the fewest motion artifacts was chosen for further evaluation. These images were transferred to a workstation for processing Volume Viewer AW4.2 (GE, Medical System, Saskatchewan, Canada). Axial, sagittal, coronary multiplanar reformations and three-dimensional images were created using standard software. Multidetected computed tomography images were analyzed by two experienced radiologists and one cardiologist.

\section{Results}

Patients' characteristics and their scan parameters are shown in Table 1 . Thirteen patients ( 8 men, 5 women; age range 31-78) who had CAFs detected previously by coronary angiography were evaulated. All patients had sinus rhythm. Heart rate of patients were ranged between 48 and $69 \mathrm{bpm}$. Six out of 13 (46\%) patients had atherosclerotic coronary artery disease. When we evaulated coronary artery segments we found a single vessel coronary artery disease in three patients, twovessel coronary artery disease in one patient, and a threevessel coronary artery disease in two patients.

In the present study, 12 out of $15(80 \%)$ CAFs originated from the left coronary artery system. In the remaining patients, CAFs originated from the RCA. Left anterior descending (LAD) coronary artery was the most common originating site of CAFs (46\%). In this study, $66 \%$ of CAFs drained into the systemic venous circulation. The pulmonary artery (PA) was the most common drainage site of CAFs (40\%). CAFs were less commonly draining into the left ventricular cavity, right ventricular outflow track (RVOT), right atrium (RA), pulmonary vein, and bronchial artery. Origins and drainage sites of CAFs are shown in Table 2.

We found double CAFs in two patients when we evaluated CAFs on the basis of coronary angiography. In one of these patients, CAFs were originating from the LAD first diagonal branch and RCA, and draining into the left ventricular cavity; in the other, CAFs were originating from the RCA and LAD second septal branch and draining into the RVOT.

When we compared images of the MDCT with the coronary angiography we found that four out of fifteen (27\%) CAFs were not visualized in the MDCT. The origins, courses, and drainage sites of fistulas were visualized by the MDCT in the remaining 11(73\%) CAFs. Unvisualized CAFs on MDCT are shown in Table 2. 
TABLE 1 Patients' characteristics and scan parameters

\begin{tabular}{|c|c|c|c|c|}
\hline Patient no. & Age/gender & Heart rate during scan, bpm & Contrast transit time, sc & $\mathrm{Z}$-axis coverage \\
\hline 1 & $62 / \mathrm{M}$ & $48 / 56$ & 24 & 90 \\
\hline 2 & $59 / \mathrm{M}$ & $64 / 66$ & 20 & 120 \\
\hline 3 & $54 / \mathrm{F}$ & $60 / 65$ & 19 & 90 \\
\hline 4 & $67 / \mathrm{M}$ & $54 / 58$ & 22 & 90 \\
\hline 5 & $64 / F$ & $62 / 68$ & 19 & 120 \\
\hline 6 & $57 / \mathrm{M}$ & $56 / 62$ & 21 & 120 \\
\hline 7 & $61 / \mathrm{M}$ & $60 / 62$ & 20 & 90 \\
\hline 8 & $71 / \mathrm{F}$ & $58 / 61$ & 22 & 120 \\
\hline 9 & $75 / \mathrm{M}$ & $64 / 68$ & 18 & 90 \\
\hline 10 & $56 / \mathrm{F}$ & $60 / 60$ & 20 & 90 \\
\hline 11 & $48 / \mathrm{M}$ & $63 / 68$ & 19 & 120 \\
\hline 12 & $70 / \mathrm{F}$ & $60 / 66$ & 19 & 90 \\
\hline 13 & $65 / \mathrm{M}$ & $65 / 69$ & 19 & 90 \\
\hline
\end{tabular}

Abbreviations: $\mathrm{bpm}=$ beat per minute; $\mathrm{F}=$ female; $\mathrm{M}=$ male; $\mathrm{sc}=$ second.

TABLE 2 Coronary artery anatomy and fistula's course of each patient detected by coronary angiography and results of MDCT

\begin{tabular}{ccccc}
\hline Patient no. & Coronary artery disease & Origin of Fistula & Drainage site of fistula & Coronary artery fistula on MDCT \\
\hline 1 & None & LAD & LVC & Detected \\
& & RCA & LVC & Detected \\
2 & Three vessels & LAD & PA & Detected detected \\
3 & Single vessel & LAD & RA & Detected \\
4 & None & LAD & LVC & Detected \\
5 & None & RCA & PA & Detected \\
6 & Single vessel & CX & PA & Not detected \\
7 & None & RCA & RVOT & Detected \\
8 & None & RVOT & Not detected \\
9 & & Reptal Branch & PA & Detected \\
10 & None & LAD & PA & Detected \\
11 & Three vessels & LAD & PA & Detected \\
13 & Two vessels & CX & PV & Detected \\
\hline
\end{tabular}

Abbreviations: $\mathrm{CX}=$ circumflex coronary artery; LAD = left anterior descending coronary artery; LVC = left ventricle cavity; $\mathrm{MDCT}=$ multidetected computed tomography; $\mathrm{PA}=$ pulmonary; $\mathrm{PV}=$ pulmonary vein; artery; $\mathrm{RA}=$ right atrium; $\mathrm{RCA}=$ right coronary artery; RVOT $=$ right ventricle outflow tract.

Percutaneous coil embolization was performed on five patients but failed in one due to the unsuitable anatomy of CAF.

The images of MDCT and coronary angiographies of four CAFs are shown in Figs. 1-4.

\section{Multidetected Computed Tomography Sensitivity}

When we evaluated images of MDCT on the basis of coronary angiography, we found overall the sensitivity of MDCT to be $73 \%$ in patients with CAFs. Furthermore, the sensitivity of MDCT was calculated as $87 \%$ in patients with fistulas coursing between coronary arteries and other vascular structures. However, the sensitivity of MDCT in patients who had CAFs coursing between coronary arteries and cardiac chambers was found to be $58 \%$.

\section{Discussion}

Here in this report, we had determined the sensitivity of MDCT in patients who had CAFs that were detected first by coronary angiography which is the "gold standard" in current literature. A few case reports of CAF diagnosed by MDCT have been published earlier. However, in the current literature, we did not find a study that included CAFs diagnosed by MDCT compared with coronary angiography, to detect accuracy or sensitivity of MDCT.

CAF is the most common form of congenital anomaly of the coronary arteries. It may be either acquired or congenital in cause. Coronary artery fistulas are commonly associated with atherosclerotic coronary artery disease. The incidence of CAF was found to be $0.18 \%$ of adult patients undergoing coronary angiography in one large series. ${ }^{2}$ Most of the coronary fistulas drain into the right 


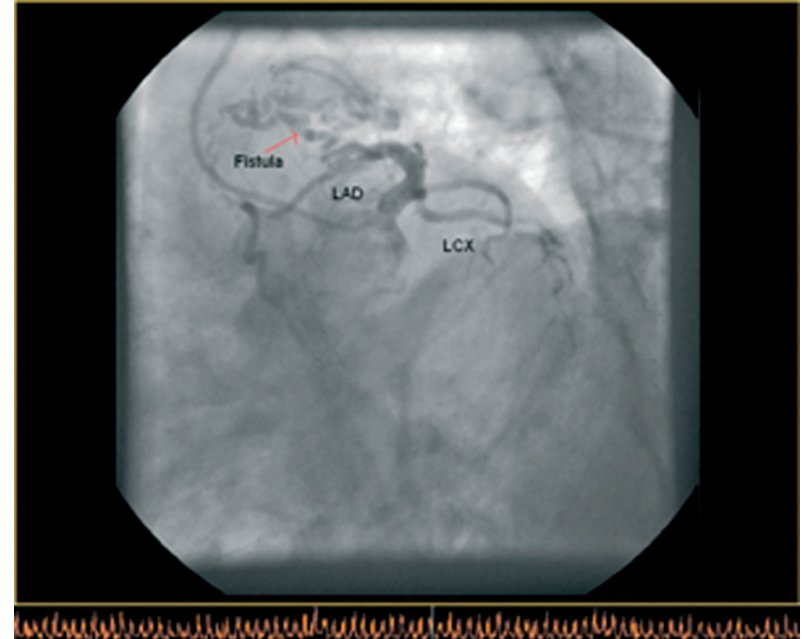

(A)

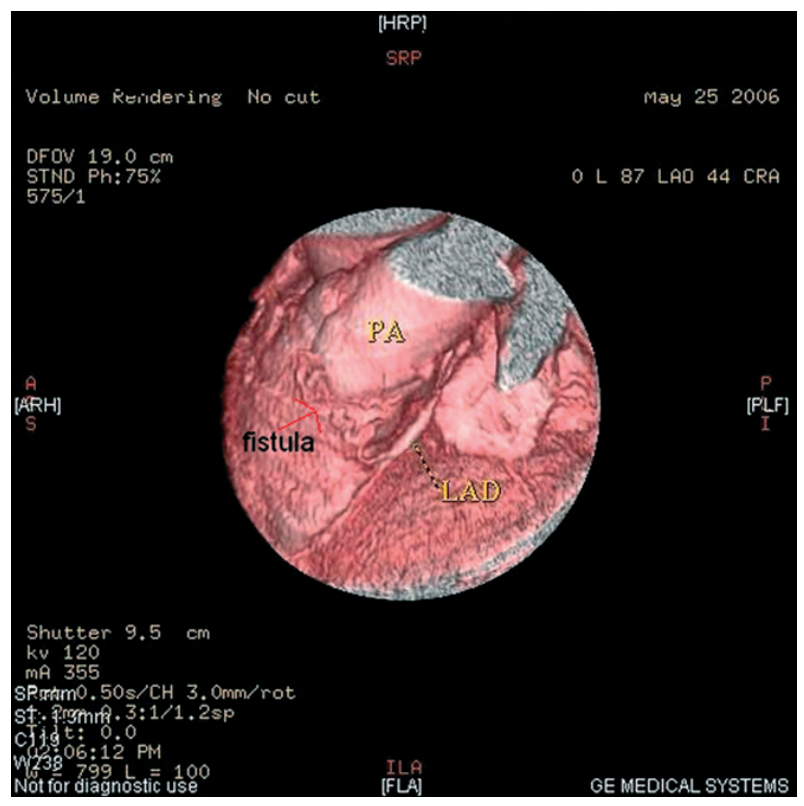

(B)

FIG. 1 (A) Coronary artery fistula originating from LAD is seen on the left anterior oblique caudal angiographic view. (B) Three-dimensional volume-rendered CT coronary angiogram shows fistula from proximal left anterior descending artery to main pulmonary artery. (LAD: Left anterior descending coronary artery; LCX: Left circumflex coronary artery).

ventricle $(41 \%)$, RA $(26 \%)$ or PA $(17 \%))^{3,5}$ Coronary sinus $(7 \%)$, left atrium $(5 \%)$, left ventricle $(3 \%)$ and superior vena cava $(1 \%)$ are less frequent drainage sites of CAFs. ${ }^{3}$ The CAFs rarely drain into pulmonary and bronchial veins.

The clinical presentation of congenital CAFs may vary considerably, depending on their anatomy and the size of the fistulous connection to the left or right side of the heart and their flow reserve. Even if a patient is initially asymptomatic, symptoms such as fatigue,

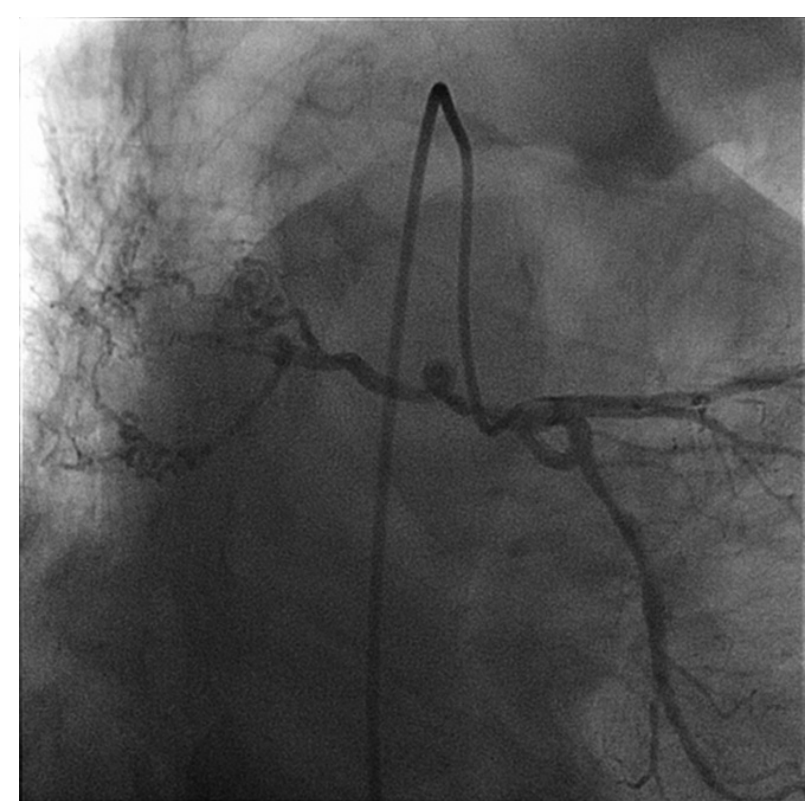

(A)

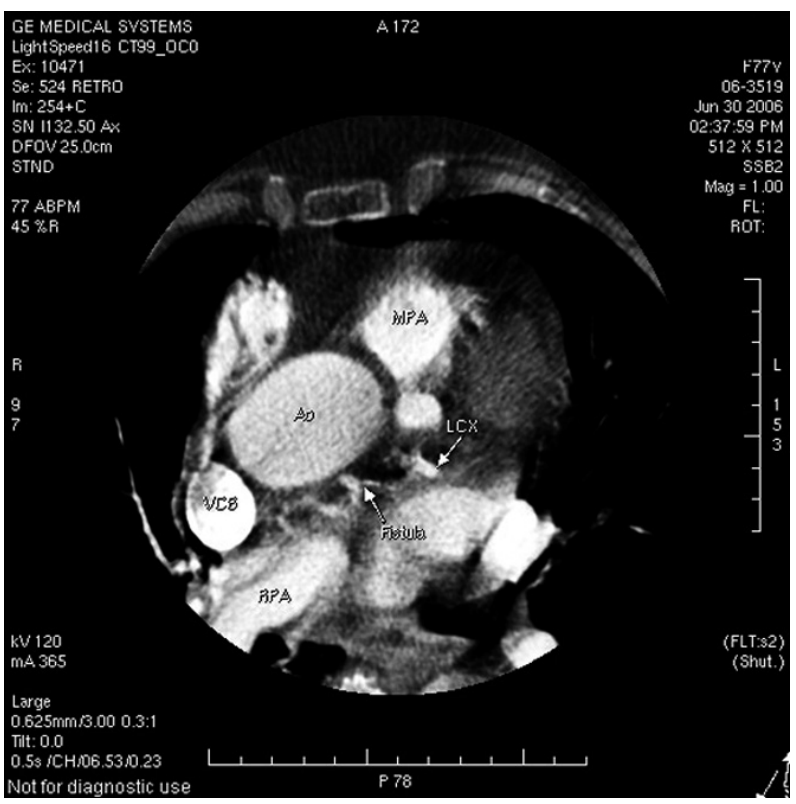

(B)

FIG. 2 (A) Anterior-posterior caudal angiographic view shows a coronary fistula between left circumflex and bronchial artery. (B) Axial imaging of MDCT shows fistula originating from the left circumflex artery draining into the bronchial artery. (LAD: Left anterior descending coronary artery; LCX: Left circumflex coronary artery; Ao: Aort; MPA: Mean pulmonary artery; RPA: Right pulmonary artery; SVC: Superior vena cava).

dyspnea, and angina are seen in the course of time. In general, symptoms are rare under the age of 20 years. Dyspnea or congestive heart failure may occur and angina pectoris is seen due to myocardial ischemia in $80 \%$ of patients over 50 years of age, who had a high 


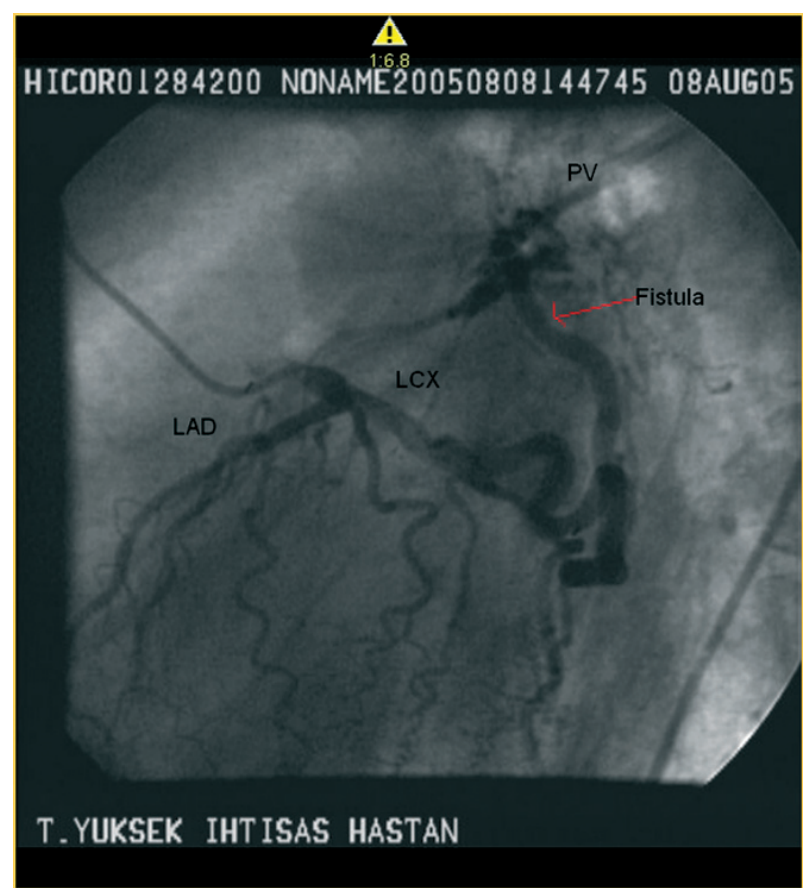

(A)

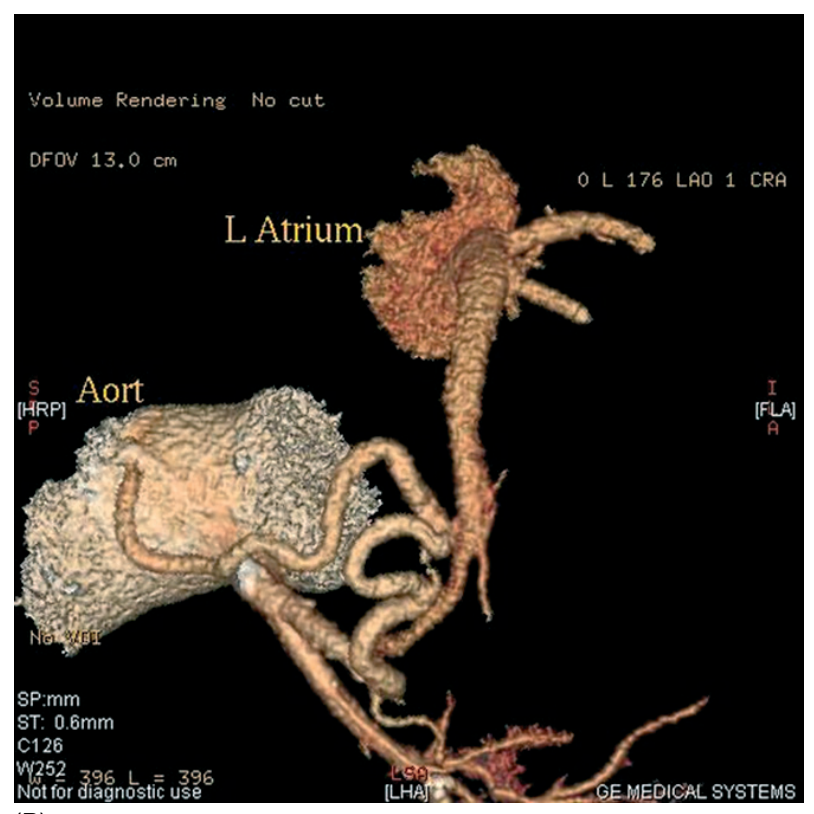

(B)

FIG. 3 (A) Left anterior oblique cranial view shows a coronary artery fistula from the left circumflex coronary artery to the pulmonary vein. (B) Three-dimensional volume-rendered CT coronary angiogram shows a fistula from the left circumflex coronary artery to the pulmonary vein. (LAD: Left anterior descending coronary artery; LCX: Left circumflex coronary artery; PV: Pulmonary vein).

flow reserve CAF. Cardiomyopathy, congestive heart failure, and atrial fibrillation can occur as late findings in patients who had CAFs. ${ }^{6}$

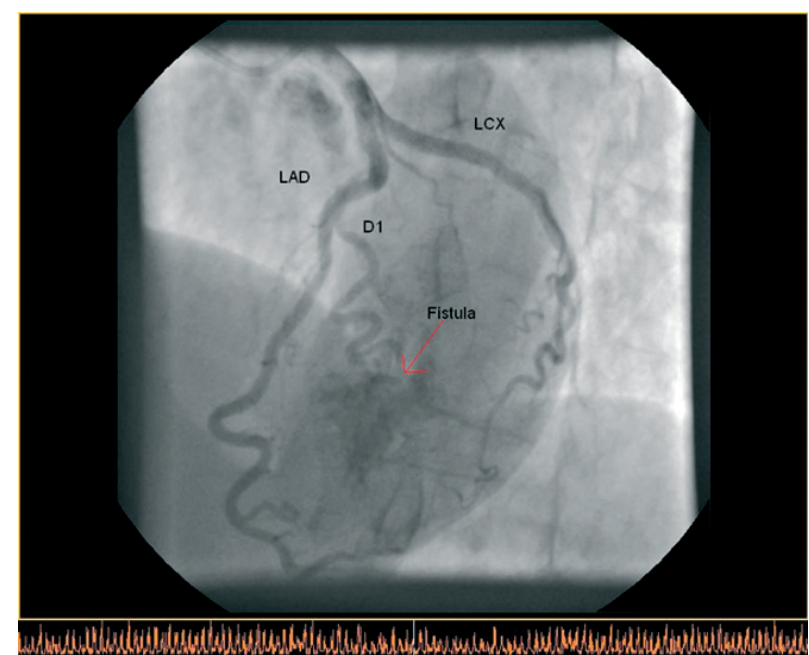

(A)

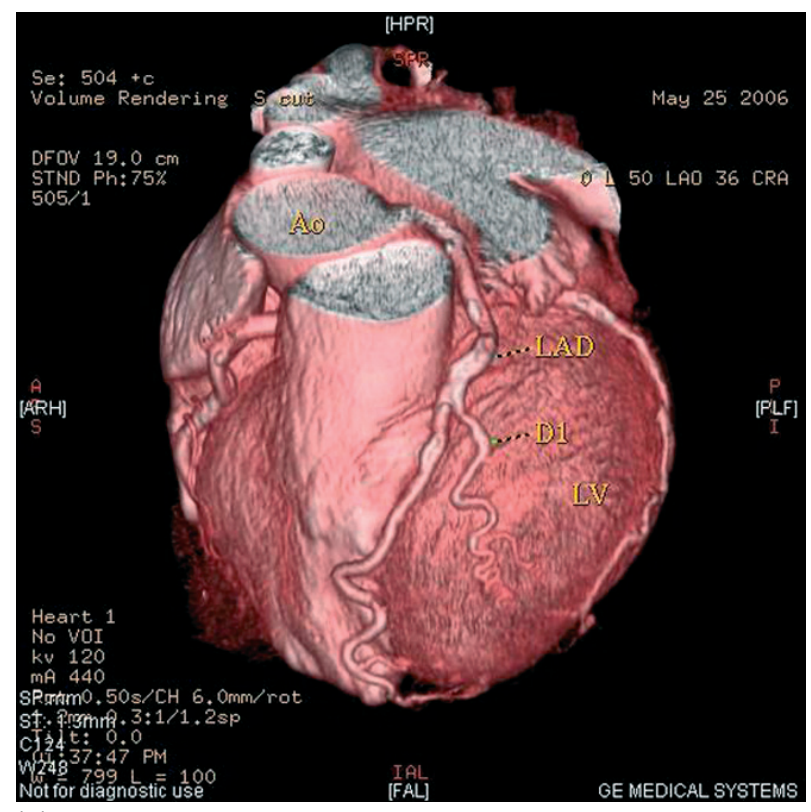

(B)

FIG. 4 (A) Left anterior oblique cranial view shows coronary artery fistula from first diagonal coronary artery to left ventricle cavity. (B) Three-dimensional volume-rendered CT coronary angiogram shows fistula from first diagonalis coronary artery to left ventricle cavity. (LAD: Left anterior descending coronary artery, LCX: Left circumflex coronary artery, D1: First diagonalis coronary artery, Ao: Aort, LV: Left ventricle).

The closure of CAFs at the time of diagnosis is recommended even in asymptomatic patients, because of perioperative morbidity and mortality increases in older patients ${ }^{7}$ and long-term results are excellent. ${ }^{8}$ Patients with CAFs which had a low flow from the coronary artery to other vascular structures or cardiac chambers have benign prognosis. Bajj et al. ${ }^{9}$ described a 51 -yearold woman with an acquired (after septal myectomy) 
coronary fistula to the left ventricle with good prognosis, in contrast to fistula to the right ventricle. Indications for fistula closure include myocardial ischemia, large leftto-right shunts, and congestive heart failure due to high flow reserve of CAF. ${ }^{10}$ Treatment of CAFs has included surgical ligation and percutaneous closure. ${ }^{11}$ The results of surgical closure are generally satisfactory provided that the fistula has a single lumen. More recently, treatment with balloon-expandable polytetrafluoroethylenecovered coronary graft stents, and electrolytically detachable platinum coils has been described. ${ }^{12}$ In our study, percutaneous coil embolization was performed successfully on four patients, but failed in one patient who had unsuitable anatomic structure for embolization of fistula.

The diagnosis of CAF can be made noninvasively with the use of transthoracic echocardiography (TTE). ${ }^{13}$ However, the accuracy of this mode of echocardiography is limited and TTE cannot view the whole course of CAF. Transesophageal echocardiograpgy (TEE) has a higher diagnostic accuracy than TTE in patients with CAF. Nekkanti et al. ${ }^{14}$ reported a case of left circumflex coronary artery fistula that color Doppler three-dimensional transesophageal echocardiography demonstrated clearly the exact site of the communication with the coronary sinus near the left atrial appendage.

MRI has become an alternative method to evaluate anatomy, flow, and function. ${ }^{15-17}$ MRI allows detailed delineation of cardiac anatomy and is useful as an additional noninvasive method in the diagnosis of CAF.

Cardiac catheterization is the gold standard in diagnosing CAFs. The origin of CAF can be clearly visualized by coronary angiography. However, the course and drainage site of the CAF are visualized less clearly by coronary angiography because of its two-dimensional imaging pattern.

MDCT provides excellent spatial resolution, which allows assessment of not only atherosclerotic coronary artery disease ${ }^{18-23}$ but also congenital coronary artery anomalies such as coronary arteriovenous fistula. ${ }^{24}$ Furthermore, MDCT can show not only an accurate anatomic relationship of CAF with adjacent structures, but also the drainage site as well. ${ }^{25}$ Clinical use of MDCT in patients with CAFs is minimal, and there is not enough data relating to this in current literature. In the present study, we used a 16-slice MDCT for detection of CAFs although it is outdated and the 64-slice MDCT is currently being used. However, we have insufficient clinical data relating to a 64-slice MDCT in patients with CAF. Nevertheless, Nakamura et al. ${ }^{26}$ reported a case of giant congenital CAF to the left brachial vein clearly detected by MDCT. They showed a usefulness of MDCT in clarifying the exact anatomy of the CAF in this case. In addition, Soon et al. ${ }^{27}$ reported how well the noninvasive CT coronary angiography with a 16-slice CT scanner characterized the anatomy of an anomalous coronary artery with a coronary cameral fistula in one case. In the present study, we determined the sensitivity of
MDCT compared with coronary angiography in patients who had CAF. Eleven of fifteen CAFs were revealed by MDCT and the overall sensitivity of MDCT was found to be $73 \%$. But, we observed an increase in sensitivity of MDCT in patients who had fistulas between two vascular structures. In these groups, a sensitivity of $87 \%$ of MDCT in patients who had CAF was found. One of the most important results of this study is that the sensitivity of MDCT was lower (58\%) in patients who had CAF coursing between coronary arteries and cardiac chambers. When we re-evaluated this population, we observed that the CAFs were small cameral vascular structures and originated from the distal segment of the coronary artery and drained into the cardiac chambers, except one CAF that was coursing between the LAD and PA.

\section{Conclusion}

Although coronary angiography is the gold standard diagnostic method for detecting the origin and course of CAFs, MDCT may be the alternative imaging method, especially in fistula coursing between the coronary artery and other vascular structures to detect fistula origin and drainage sites because of its excellent spatial resolution and ability to show the relationship of anatomic structures. In this population, accuracy or sensitivity of MDCT is quite high. However, MDCT cannot be a suitable imaging method in patients who have CAFs originating in the distal segment of a coronary artery with small vascular structure and coursing between coronary arteries and cardiac chambers. Our study is important because it is the first such study in current literature. However, we need a large-scale study to provide more reliable sensitivity levels of MDCT in patients with CAF.

\section{Study Limitations}

Having evaluated 13 patients with a total of 15 coronary artery fistulas, the current numbers are too low to determine the exact sensitivity of MDCT compared with coronary angiography. Therefore, we need a larger number of subjects to obtain the true sensitivity of MDCT. Suitable heart rates must be maintained during the MDCT procedure, especially in patients who have small cameral and low flow to detect the whole course of CAF.

\section{References}

1. Robert WC: Major anomalies of coronary arterial origin seen in adulthood. Am Heart J 1986;111:941-963

2. Yamanaka O, Hobbs RE: Coronary artery anomalies in 126,595 patients undergoing coronary arteriography. Cathet Cardiovasc Diagn 1990;21:28-40

3. Lin FC, Chang HJ, Wen MS, Wu D: Multiplane transesophageal echocardiography in the diagnosis on congenital coronary artery fistula. Am Heart J 1995; 130:1236-1242 
4. Wilcox WD, Neal MJ, Alpert BS, Taylor AB, Dooley KJ: Localized occurrence of congenital coronary artery fistula in the southeast United States. Am J Cardiol 1986;57:361-363

5. Gandy KL, Rebeiz AG, Wang A, Jaggers JJ: Left main coronary artery-to-pulmonary artery fistula with severe aneurysmal dilatation. Ann Thorac Surg 2004;77:1081-1083

6. Perloff JK. Congenital coronary artery fistula In: Perloff JK, ed. The Clinical Recognition of Congenital Heart Disease. Philadelphia, PA: Saunders; 1994; 562-580

7. Schumacher G, Roithmaier A, Lorenz HP, Meisner H, Sauer U, et al.: Coronary artery fistula in infancy and childhood: diagnostic and therapeutic aspects. Thorac Cardiovasc Surg 1997;45:287-294

8. Cheung DL, Au WK, Cheung HH, Chiu CS, Lee WT: Coronary artery fistulas: long-term results of surgical correction. Ann Thorac Surg 2001;71:190-195

9. Bax JJ, Raphael D, Bernard X, Vanoverschelde JL: Echocardiographic detection and long-term outcome of coronary artery-left ventricle fistula after septal myectomy in hypertrophic obstructive cardiomyopathy. J Am Soc Echocardiogr 2001;14(4):308-310

10. Lai MC, Chen WJ, Chiang CW, Ko YL: An unusual case of dual coronary artery fistulas to main pulmonary artery. Chang Gung Med J 2002;25:51-55

11. Kamiya H, Yasuda T, Nagamine H, Sakakibara N, Nishida S, et al.: Surgical treatment of congenital coronary artery fistulas: 27 years' experience and a review of the literature. J Cardiol Surg 2002;17:173-177

12. Goldberg SL, Makkar R, Duckwiler G: New strategies in the percutaneous management of coronary artery fistulas: a case report. Catheter Cardiovasc Interv 2004;61:227-232

13. Yang Y, Li Z, Wang X: Assessment of coronary artery fistula, by color Doppler echocardiography. Echocardiography 1998;15:67-72

14. Nekkanti R, Mukhtar O, Nanda NC, McGiffin DC: Transesophageal color Doppler three-dimensional echocardiographic assessment of left circumflex coronary artery fistula. Echocardiography 2002;19(7 Pt 1):573-575

15. Boxer RA, LaCorte MA, Singh S, Ishmael R, Cooper R, et al.: Noninvasive diagnosis of congenital left coronary artery to right ventricle fistula by nuclear magnetic resonance imaging. Pediatr Cardiol 1989;10:45-47

16. Kubota S, Suzuki T, Murata K: Cine magnetic resonance imaging for diagnosis of right coronary arterial-ventricular fistula. Chest 1991;100:735-737
17. Duerinckx AJ, Shaaban A, Lewis A, Perloff J, Laks H: 3D MR imaging of coronary arteriovenous fistulas. Eur Radiol 2000; 10:1459-1463

18. Achenbach S, Giesler T, Ropers D, Ulzheimer S, Derlien H, et al.: Detection of coronary artery stenoses by contrast-enhanced, retrospectively electrocardiographically-gated, multislice spiral computed tomography. Circulation 2001;103:2535-2538

19. Schroeder S, Kopp AF, Baumbach A, Meisner C, Kuettner A, et al.: Noninvasive detection and evaluation of atherosclerotic coronary plaques withy multislice computed tomography. J Am Coll Cardiol 2001;37:1430-1435

20. Sato $\mathrm{Y}$, Matsumoto $\mathrm{N}$, Kato $\mathrm{M}$, Inoue $\mathrm{F}$, Horie $\mathrm{T}$, et al.: Noninvasive assessment of coronary artery disease by multislice spiral computed tomography using a new retrospectively ECG-gated image reconstruction technique: comparison with angiographic results. Circ J 2003;67:401-405

21. Achenbach S, Ropers D, Hoffmann U, MacNeill B, Baum U, et al.: Assessment of coronary remodeling in stenotic and nonstenotic coronary atherosclerotic lesions by multidetector spiral computed tomography. J Am Coll Cardiol 2004;43:842-847

22. Inoue F, Sato $\mathrm{Y}$, Tani S, Uchiyama $\mathrm{T}$ : Evaluation of plaque texture by multislice computed tomography in patients with acute coronary syndrome and stable angina. Circ J 2004;68: 400-404

23. Imazeki $\mathrm{T}$, Sato $\mathrm{Y}$, Inoue $\mathrm{F}$, Anazawa $\mathrm{T}$, Tani $\mathrm{S}$, et al.: Evaluation of coronary artery remodeling in in patients with acute coronary syndrome and stable angina by multislice computed tomography. Circ $J$ 2004;68:1045-1050

24. Sato Y, Mitsui M, Takahashi H, Miyazawa T, Okabe H, et al.: A giant left circumflex coronary artery-rihgt atrium arteriovenous fistula detected by multislice spiral computed tomography. Heart Vessels 2003; 19:55-56

25. Tan KT, Chanberlain-Webber R, McGann G: Characterisation of coronary artery fistula by multi-slice computed tomography. Int $J$ Cardiol 2006;112(2):311-312

26. Nakamura M, Matsuoka H, Kawakami H, Komatsu J, Itou $\mathrm{T}$, et al: Giant congenital coronary artery fistula to left brachial vein clearly detected by multidetector computed tomography. Circ J 2006;70(6):796-799

27. Soon KH, Selvanayagam J, Bell KW, Tang SH, Pereira J, et al.: Giant single coronary system with coronary cameral fistula diagnosed on MSCT. Int J Cardiol 2006;106(2):276-278

\section{INDEX TO ADVERTISERS}

Actos Family.

AstraZeneca Crestor.

Boehringer-Ingelheim Micardis............................................................................... $1-2$

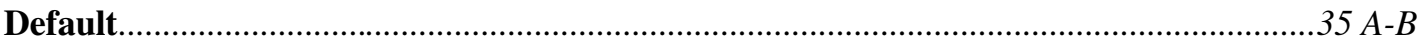

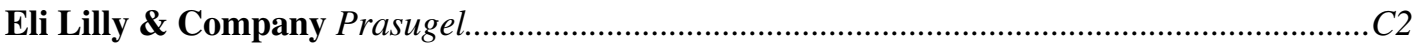

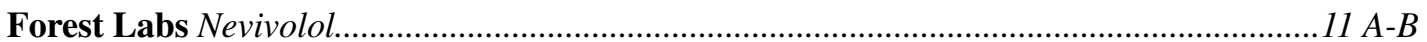

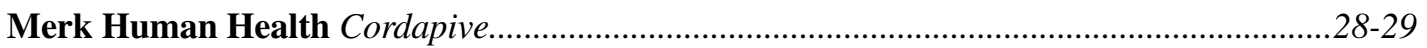

Nitromed Bidil.

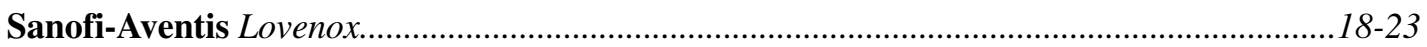

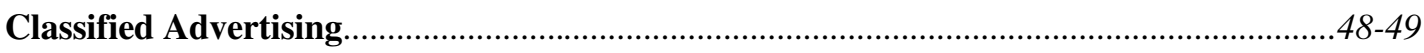

This index is provided as a service. Every effort to achieve accuracy is made. Publisher is not responsible for errors or omissions 